\title{
Characterization of a probiotic Lactobacillus fermentum isolated from snakehead, channa striatus, stomach
}

\begin{abstract}
This study sought to isolate and characterize lactic acid bacteria (LAB) from stomach of adult snakehead fish, Channa striatus, to be used as probiotics for freshwater fish. A total of 13 strains were isolated from the stomach of 10 fish, and 4 of these belonged to LAB. Strain LAB-3 showing highest in vitro growth inhibition of Aeromonas hydrophila in a disk diffusion test was identified as Lactobacillus fermentum by conventional and molecular techniques and evaluated in vitro through various tests. The bacterium could grow at $\mathrm{pH} 3-8$; but the optimum growth was observed at $\mathrm{pH}$ 6. Moreover, LAB-3 grew at 0.15 and $0.3 \%$ bile salt concentrations, from 15 to $45 \mathrm{C}$, and at $4 \% \mathrm{NaCl}$. L. fermentum showed in vitro inhibitory activity against three fish pathogens, A. hydrophila, Pseudomonas aeruginosa, and Shewanella putrefaciens, tested by disk diffusion and well diffusion methods. Antibiotic sensitivity tests indicated that L. fermentum was resistant to streptomycin, gentamycin, and kanamycin, intermediate to tetracycline, but sensitive to chloramphenicol, amoxicillin, and ampicillin. Challenge test by using A. hydrophila showed that survival of snakehead was significantly $(\mathrm{P}<0.05)$ improved when $2 \times 106 \mathrm{LAB}-3 / \mathrm{g}$ was supplemented to the diet. Therefore, this study suggests that L. fermentum might be a promising probiotic in snakehead aquaculture.
\end{abstract}

Keyword: Probiotic; Lactobacillus fermentum; Lactic acid bacteria; Snakehead fish; Channa striatus. 